\title{
Pérez Juste, R., Galán González, A. \& Quintanal Díaz, J. (2012). Métodos y diseños de investigación en educación. Madrid: UNED. 599 pp.
}

\author{
Anibal Meza Borja ${ }^{1 \mathrm{a}}$ \\ ${ }^{1}$ Universidad Nacional Mayor de San Marcos, Lima, Perú. \\ ${ }^{a}$ Doctor en Psicología con especialidad en Psicología Educativa. Profesor principal de la Facul- \\ tad de Psicología de la Universidad Nacional Mayor de San Marcos.
}

Recibido: $21-06-13$

Aprobado: $27-08-13$

*Correspondencia

Email: amezaborja@gmail.com

\section{Citar Como:}

Meza Borja, A. (2013). Métodos y diseños de investigación en educación. Madrid: UNED. 599 pp. Pérez Juste, R., Galán González, A. \& Quintanal Díaz, J. (2012). Propósitos y Representaciones, l(1), 173-183. doi: http:// dx.doi.org/10.20511/pyr2013.v1n1.14 
Se dice que una de las funciones esenciales de la universidad debiera ser la producción de conocimientos, y que la actividad generadora de los mismos es la investigación científica. De ahí que su enseñanza constituya tanto una necesidad como una obligación en los programas de formación académica en todas las disciplinas y las carreras profesionales. Los estudiantes universitarios, además de profesores que lo introduzcan en los conocimientos conceptuales y procedimentales sobre investigación, particularmente, requieren de textos y manuales serios y rigurosos que les permitan profundizar sus conocimientos sobre los diversos aspectos de la investigación. Es en ese sentido que a los ya clásicos libros sobre investigación en el campo de la educación de Ary, Jacobs y Razavieh (1989), Best (1982), McMillan y Schumacher (2006) y Travers (1986), habría que agregar el que aquí se reseña.

Los autores: Ramón Pérez Juste es conocido por su amplia producción de artículos y libros en temas relacionados a la calidad de la educación, evaluación de programas educativos, estadística, pedagogía experimental, y métodos y diseños de investigación en el ámbito de la educación. El libro que reseñamos está publicado en coautoría con Arturo Galán González (investigador en temas relacionados a la calidad educativa, el clima de trabajo, la evaluación de los centros educativos y de la competencia docente en la educación superior) y José Quintanal Díaz (interesado muy vivamente por temas en educación infantil y de la lectura en ese nivel educativo).

El libro: Métodos y diseños de investigación en educación, es un manual universitario. Es en ese sentido que sus temas se organizan en unidades didácticas (U. D.), cada una de las cuales comprende la enunciación del tema a tratar, una presentación, una introducción y el desarrollo de los tópicos del caso. Las unidades didácticas I y III desarrollan tres temas, cada una y la unidad II, ocho, haciendo un total de 14 temas. Al final del desarrollo de cada tema se ofrece un resumen.

En la primera U. D. los autores discuten de modo enjundioso conceptos como los de ciencia, método, paradigmas de investigación y métodos cuantitativos y cualitativos. En recuadros que hacen atractiva la diagramación del libro, transcriben de primera mano citas de autores muy prestigiosos en los campos de la filosofía (Descartes, Dewey, Kuhn, entre otros) y de la investigación científica (Bunge, Kerlinger, Nagel, Popper, 
etc.) y comentan el sentido de esas citas. En el tema uno se caracteriza la investigación científica como un procedimiento para obtener conocimiento. Se discute la condición de la educación como objeto de conocimiento, para lo cual primero se describen y analizan los rasgos del método general de la ciencia y luego se hace referencia a la distinción entre ciencias naturales (en las que hay una "distancia" entre el investigador y el objeto investigado) y las ciencias sociales (en la que hay una interacción entre el investigador y la realidad estudiada). Cuando desarrollan la idea de la educación como ámbito disciplinar, plantean que si bien la educación es un arte puede beneficiarse de conocimientos obtenidos mediante el uso del método científico. Por lo que la disciplina que utiliza el método científico debe aspirar a constituirse como una ciencia. Además, sostienen que la educación es un "ámbito del saber" tan amplio (establecen una analogía con la medicina) que sería impensable llegar a "una disciplina unitaria, homogénea en sus planteamientos" (p. 35).

Al abordar el problema de los paradigmas, se menciona, entre otros, a Khun, para quien al no existir un paradigma único predominante en las ciencias sociales (entre las cuales estaría la educación) éstas resultarían preparadigmáticas, condición que lejos de ser adversa para el desarrollo de esas ciencias, resultaría favorable dada la amplia gama de asuntos que deben investigar. Se señala que, en el ámbito de las ciencias sociales, los paradigmas opcionales serían el positivista (cuantitativo) y naturalista (cualitativo), al que podría agregarse el paradigma crítico (orientado a la transformación de la sociedad). Luego se hacen una caracterización y valoración de los paradigmas, tomando los planteamientos de Cook y Reichardt, y también se establecen las diferencias entre los paradigmas asumiendo las ideas de Lincoln y Guba (tabla 1).

Tabla 1.

Supuestos en torno a los paradigmas cuantitativo y cualitativo

\begin{tabular}{lccc}
\hline Paradigmas & Ontológicos & $\begin{array}{c}\text { Supuestos } \\
\text { Epistemológicos }\end{array}$ & Metodológicos \\
\hline Cuantitativo & Realismo & Objetivismo & Intervencionismo \\
Cualitativo & Relativismo & Subjetivismo & Hermenéutica \\
\hline
\end{tabular}

Los autores advierten que las consideraciones que se reflejan en la tabla 1 podrían llevar a la idea que los paradigmas son polares $\mathrm{y}$, por 
tanto, irreconciliables y que no sería posible concebir que alguien estuviera interesado, al mismo tiempo, por explicar y comprender un fenómeno determinado. Señalan, siguiendo a Cook y Reichardt, que la elección del método debe depender de "las exigencias de la situación de investigación de que se trate" (p. 43), sugiriendo una complementariedad entre ambos paradigmas y sus métodos (cosa que ocurre particularmente en la investigación evaluativa). El tema uno se cierra con la derivación de una justificación de la necesidad de la investigación en educación, a pesar de la complejidad del hecho educativo. Investigación abierta a la diversidad metodológica, y de ser mejor con una perspectiva de complementariedad de los paradigmas investigativos.

En el tema dos, luego de señalar las funciones de la ciencia (predictiva y de control), y caracterizar el conocimiento científico por su precisión, su enfoque causalista, y su objetividad, se destaca la importancia del método. Particularmente, el método hipotético-deductivo-experimental es resumido a partir de la propuesta de Dewey: percatamiento de un problema, identificación y definición del mismo, planteamiento de hipótesis; consecuencias, si es que fueran ciertas las hipótesis planteadas, las cuales deben ser formuladas con precisión en la investigación experimental, en forma operativa, y la validación de las hipótesis mediante la prueba pertinente. El método descrito es una síntesis de observación y razonamiento, de deducción e inducción, de análisis y de síntesis, que siguieron científicos como Newton y Galileo, entre otros. Los autores hacen notar que sobre ese método científico (general), los problemas particulares de cada ciencia demandan técnicas específicas, que dependen del desarrollo de cada ámbito disciplinar.

El proceso y la lógica de la investigación científica tienen en el libro una presentación relativamente amplia. El problema, dicen los autores, "no se revela a cualquiera sino a quien se interesa por los problemas y tiene una adecuada formación., conoce el campo de que se trate y domina adecuadamente las técnicas apropiadas" (p. 63). Comentaríamos que, particularmente es el caso de los hallazgos serendípicos en ciencia. Señalan ciertas condiciones para que un problema sea científico: que sea resoluble, incrustado en una teoría, que derive en un conjunto de otros problemas, formulado en forma clara y precisa, que permita derivar una hipótesis, que oriente la selección de métodos y procedimientos que llevarán a su resolución. 
Con relación a la hipótesis y su validación, se señala que la formulación de hipótesis se constituye en un aspecto crucial en la resolución de problemas, dado que supone hacer conjeturas sobre la relación de las variables, y orienta en la selección de procedimientos e instrumentos para recoger los datos y el tipo de prueba a que debe ser sometida la hipótesis. Ninguna hipótesis es contrastable por sí misma, para serlo ha de tener consecuencias observables y la traducción de la hipótesis a estas consecuencias se conoce como operativización u operacionalización. El asunto de la validación de hipótesis lleva a discutir los siguientes tópicos: muestra y muestreo, elección del diseño de investigación para el control de las variables extrañas, elección de procedimientos de medición y nivel de medición, y elección de pruebas estadísticas.

Luego de caracterizar el conocimiento científico experimental, como basado en la observación y la experimentación científicas, que recurre a la medición, el control y la verificabilidad intersubjetiva, se discuten las posibilidades y limitaciones de la aplicabilidad de la metodología científico-experimental a la educación: posibilidades del establecimiento de leyes generales, de la medición y la evaluación, de control y de la verificabilidad intersubjetiva. Se termina el tema dos con un examen de las repercusiones metodológicas acerca de la validez y la confiabilidad de las medidas que conducen al investigador a conocer el margen de error que afecta a sus resultados. Otros asuntos metodológicos están referidos a la selección de las muestras (su suficiencia y representatividad) y la selección de un diseño adecuado de investigación para el control de las variables extrañas.

El tema tres gira en torno al informe de investigación y su relación con la calidad de ésta. Se discute el valor, sentido y utilidad del informe, sus elementos, su tipología (en función de la perspectiva de aproximación: cuantitativa, cualitativa o evaluativa). Se enfatiza en que el informe debe reflejar la calidad de la investigación y se señalan los criterios que permitirían juzgarla: en relación a los objetivos (pertinencia, novedad y factibilidad), en relación a los procesos (transparencia, sistematización de sus fases de desarrollo), y en relación a los resultados (explotación de los resultados y maximización del impacto científico). 
La U. D. II cubre ocho temas. Todos relacionados con el asunto de los diseños de investigación (diseños per se y las pruebas estadísticas en función de los diseños de investigación).

El tema cuatro desarrolla el concepto de diseño, sus objetivos (de los cuales uno esencial es el control de la varianza, que depende de la validez interna de la investigación o el control de la variable independiente -se hace referencia aquí a un principio formulado por Kerlinger: Max-Min-Con), sus tipos: pre-experimentales, experimentales, cuasi-experimentales); y los criterios para la selección de un diseño. Se reflexiona acerca de la pertinencia de los diseños de investigación en el ámbito de la educación.

El asunto central del tema cinco es el experimento: su definición, sus características, sus limitaciones en el campo de la educación, la validez de los diseños y los factores de invalidez interna y externa de los diseños. Frente a las limitaciones de los diseños experimentales en educación se destacan las bondades de la investigación ex-post-facto, de la cual se dan la definición, sus características, sus modalidades (estudios de campo, transversales y longitudinales, los de casos).

El tema seis presenta los diseños de dos grupos, los que a juicio de los autores son los de más uso en la investigación cuantitativa en el campo educativo. Se discute la noción de control y las formas de su aseguramiento mediante los diseños, presentándose los principales diseños de dos grupos (con sólo post test, con pre-test, con sujetos independientes o emparejados), haciéndose referencia a las pruebas estadísticas pertinentes. Adicionalmente se presentan los diseños factoriales, de cuadrado latino y el de covarianza.

En el tema siete el libro focaliza en los conceptos de inferencia estadística (haciéndose mención a las nociones de población, muestra y medición), distribución muestral y estimación de parámetros, y se los vincula con los diseños de uno y dos grupos. La inferencia es concebida como un procedimiento de extracción de conclusiones, a partir del análisis de los datos recogidos de una muestra (estimándose parámetros y contrastando hipótesis). Luego de enumerar las propiedades de los "estimadores", se presenta con algún detalle los procedimientos de estimación de algunos parámetros: media aritmética, correlación de Pearson, diferencia de medias. Este tema se termina con la discusión de lo que es el contraste de hipótesis. 
En el tema ocho el asunto esencial es el contraste de hipótesis. Luego de hablar acerca del sentido de la prueba de significación de la hipótesis nula, se indican los pasos que según Warner han de seguirse en el proceso de realizar la prueba de significación de la hipótesis nula. Los resultados del procedimiento pueden llevar a aceptar o rechazar la hipótesis nula, dando lugar a cometer errores de tipo I (la probabilidad de rechazar la hipótesis nula cuando ésta es verdadera) y de tipo II (no rechazar la hipótesis nula cuando ésta es falsa). Este tema continúa con la presentación de los pasos que hay que seguir para la contrastación de hipótesis (selección de una muestra aleatoria, formulación de las hipótesis estadísticas - nula y alternativa-, elección del nivel de significación, determinación de la distribución muestral y la región de rechazo, y utilización del valor exacto de la probabilidad asociada al estadístico de contraste). Se cierra este tema señalando los errores frecuentes en la utilización e interpretación de los resultados de la prueba de significación de la hipótesis nula y la significación estadística del tamaño del efecto.

El tema nueve presenta y desarrolla los conceptos y procedimientos para el uso de pruebas paramétricas, aplicables a diferentes diseños de investigación de dos grupos. Así, se revisan las pruebas $\boldsymbol{t}$ y $\boldsymbol{z}$ (se ofrecen ejemplos $\mathrm{y}$, en forma muy didáctica, las nociones y procedimientos estadísticos pertinentes para su aplicación). Se propone como alternativa a éstas, la prueba $\mathbf{F}$ (análisis de varianza ANAVA o ANOVA, según se le llame castizamente o con referencia a su denominación en inglés). Las pruebas señaladas tanto para muestras independientes como muestras relacionadas.

El tema 10 está dedicado a la presentación de algunas (de las muchas pruebas, para medidas nominales u ordinales) pruebas no paramétricas. Como en el caso de las pruebas paramétricas, aquí también se ofrecen las nociones estadísticas y los procedimientos pertinentes. Las pruebas revisadas son $J i$ cuadrada y U de Mann Whitney (para grupos con sujetos seleccionados y asignados al azar) y las pruebas de los signos y la $\mathrm{T}$ de Willcoxon (para el caso de medidas ordinales).

El último tema, en 11, de la segunda unidad didáctica presenta y desarrolla pruebas paramétricas para diseños con más de dos grupos (tres tratamientos, tres niveles de una variable, etc.), para los diseños con grupos naturales o "intactos", "bloques" de sujetos lo más homogéneos posible - 
diseños de cuadro latino-; diseños con dos o más variables independientes actuando sobre una misma variable dependiente o diseños factoriales. Las pruebas a usarse en las circunstancias mencionadas son en realidad variantes de la prueba F, con las adecuaciones que cada caso demanda. En el libro se ofrece un anexo a la U. D. II, en el cual se pretende ilustrar la elaboración de un informe de investigación con sus diferentes elementos, a partir de un caso concreto de investigación: introducción (identificación del problema), la formulación de hipótesis, la definición de las variables de la investigación, la elección de un diseño, determinación de una muestra, elección del instrumento y la descripción de procedimientos; la presentación de los resultados; el tratamiento estadístico de los datos y la interpretación de los mismos, y la presentación de conclusiones. Luego se realiza un "enjuiciamiento" de la investigación, en función de si cumple ciertos criterios aplicados a los elementos del informe, y especialmente se examinan la validez interna y externa de la investigación, así como el rigor y la pertinencia del tratamiento estadístico de los datos.

Los tres temas de la U. D. III están dedicados a presentar los aspectos esenciales de la metodología cualitativa de la investigación.

El tema 12 se centra en su caracterización, sus alcances y sus límites, el proceso de la investigación cualitativa, desde la propuesta inicial, el trabajo de campo, el análisis y la interpretación de los datos; así como aspectos fundamentales en la elaboración del informe y la derivación de líneas de acción. Se discute la pertinencia, las bondades y limitaciones de la investigación cualitativa en ámbitos educacionales.

El tema 13 focaliza en los aspectos metodológicos y técnicos para la recogida de datos de carácter cualitativo, como por ejemplo la observación participante en situaciones naturales, que supone la realización de las siguientes acciones: seleccionar un problema, diseñar la investigación, seleccionar el escenario y acceder a él, la selección de informantes, esclarecer y definir roles, recoger la información, registrarla, elaborar y analizar la información y la redacción del informe. Otra técnica presentada es la entrevista cualitativa o en profundidad, las acciones que supone la técnica son: seleccionar a los entrevistados, establecimiento del rapport (o nivel de confianza), realización de las entrevistas, registro meticuloso de las entrevistas, analizar la información y elaborar las conclusiones. 
Las técnicas narrativas son presentadas en el tema 13, la más típica de este tipo de técnicas es la lectura de documentos (cartas, diarios, biografías, autobiografías, actas, etc.). Estos documentos pueden ser personales o institucionales.

El tema 14, el último de los temas, presenta concepciones acerca del sentido que se les puede dar a los datos en una investigación cualitativa: codificación, indexación o recuperación de la información, o la confirmación de conceptos o teorías. En el libro se agrupan las técnicas cualitativas en tres grupos de procedimientos: los de generación teórica (exploración, descripción, interpretación, teorización), de expansión y contrastación de hipótesis o teorías (muestreo teórico, casos discrepantes, casos negativos), y analítico-manipulativos (reducción de datos, exposición de datos, elaboración y verificación de conclusiones). Se desarrolla con alguna extensión el procesamiento analítico de datos en la investigación cualitativa, el cual supone la realización de ciertas tareas como la reducción de datos (separación de unidades de análisis, identificación, clasificación y agrupamiento), disposición de datos (transformación simbólica), obtención y validación de conclusiones (extracción de conclusiones y validación).

En la reducción de datos, se procede separando la información original en partes utilizando criterios temporales, espaciales, gramaticales o temáticos, luego que los datos son segmentados deben dar lugar a categorizaciones. La categorización y codificación de los datos llevará a una identificación de los contenidos y su clasificación en unidades distinguibles. El proceso termina en la síntesis o agrupamiento de la información asignada a cada una de las categorías establecidas. En la disposición de los datos se trata de juzgar y evaluar similitudes conceptuales, afinar el poder discriminatorio de las categorías y descubrir patrones en los hechos o las conceptualizaciones, todo lo cual demanda una alta competencia intelectual, que supone también una gran creatividad para organizar los datos mediante recursos gráficos, por ejemplo.

La obtención y validación de conclusiones supone estar en condiciones de aportar información sistemática y coherente, que de respaldo a determinados enunciados y lugar a la argumentación. Lo que va a dar respaldo a los hallazgos de la investigación es el proceso de triangulación, el debate con 
los pares, el juicio de los sujetos investigados, o la búsqueda de evidencia en contra. Una acumulación de datos por la simple acumulación de los mismos no lleva a nada, lo que cabe es que ella dé lugar a la elaboración de una teoría explicativa de los hechos reflejados en tales datos. El proceso de una investigación cualitativa se reflejaría en la enumeración de los siguientes pasos: definir el fenómeno a investigar, formular una hipótesis explicativa del mismo, estudiar un caso a fin de apreciar si tal hipótesis es adecuada o no, reformular la hipótesis en el caso que no explique el fenómeno estudiado, buscar casos que refuten la hipótesis, reformular la hipótesis en caso de hallar casos negativos y continuar el proceso hasta que la hipótesis haya sido plenamente puesta a prueba.

Se finaliza el tema ofreciendo un conjunto de recursos para el tratamiento informatizado de los datos en investigaciones cualitativas. Los programas que se presenta muy sumariamente, entre otros, son: AQUAD (Analysys of Qualitative Data), útil para las tareas de codificación a fin de permitir la reducción, presentación y extracción o elaboración de conclusiones; NUD.IST (Non-numerical Unstructured Data * Indexing Searching and Theorizing), útil para segmentar y organizar los datos de la investigación, asignar categorías, establecer relaciones entre las categorías, realizar búsquedas textuales específicas, y construir matrices y tablas de frecuencias con la información importante; QSR NVIVO, un programa opcional al anterior, con el cual es posible gestionar datos y textos enriquecidos, usando recursos de edición como negrita, cursiva, colores, etc.; WinMAX, diseñado para facilitar el análisis de contenido de un texto, permite, entre otras cosas, visualizar funciones básicas como códigos y memos, codificación compleja $\mathrm{y}$ flexible, y funciones de recuperación, búsqueda lexical y codificación automática, importar y exportar matrices de datos, etc.; MAXQDA, que es una versión de WinMAX; Atlas.ti (Qualitative data analysys), un programa muy potente para el análisis visual de datos cualitativos de textos, gráficos, audio y video, el programa ayuda a descubrir fenómenos complejos que se encuentran "ocultos" en los datos cualitativos.

Treinta páginas del libro están dedicadas a un glosario, que de por sí aumentan el valor instructivo y didáctico de este.

Para terminar. Volvemos sobre las primeras líneas de esta reseña y queremos decir que con el libro de Juste, Galán y Quintanal, se enriquece 
muy considerablemente la bibliografía sobre investigación en educación en nuestro medio, y debe constituir un material de enseñanza para estudiantes de pregrado y aún de postgrado. Sus méritos son: hacer una presentación relativamente amplia de sus temas, ofrecer ejemplos que aclaran tanto asuntos conceptuales como de procedimiento; presentar los asuntos estadísticos con la suficiente amplitud y claridad; y apoyarse en una amplia y rigurosa bibliografía, que permite a los autores hacer citas valiosas y luego comentarlas de manera beneficiosa para el lector.

\section{Referencias}

Ary, D., Jacobs, A. \& Razavieh. (1989). Introducción a la investigación pedagógica. $2 \mathrm{a}$ ed. México D. F.: Trillas.

Best, J. (1982). Cómo investigar en educación. 9a ed. Madrid: Morata.

McMillan, J. \& Schumacher, S. (2006). Investigación educativa. Una introducción conceptual. Madrid: Pearson.

Travers, R. (1,986). Introducción a la investigación educacional. Barcelona: Paidós. 